\title{
Neuropsychiatric symptoms in a European Huntington's disease cohort (REGISTRY)
}

\author{
Erik van Duijn, ${ }^{1,2}$ David Craufurd, ${ }^{3,4}$ Anna A M Hubers, ${ }^{1}$ Erik J Giltay, ${ }^{1}$ \\ Raphael Bonelli, ${ }^{5}$ Hugh Rickards, ${ }^{6}$ Karen E Anderson, ${ }^{7}$ Marleen R van Walsem, 8,9 \\ Rose $\mathrm{C}$ van der Mast, ${ }^{1}$ Michael Orth, ${ }^{10} \mathrm{G}$ Bernhard Landwehrmeyer, ${ }^{10}$ the European \\ Huntington's Disease Network Behavioural Phenotype Working Group
}

For numbered affiliations see end of article.

\section{Correspondence to}

Dr Erik van Duijn, Department of Psychiatry, Leiden University Medical Centre, PO Box 9600, Leiden 2300 RC,

The Netherlands;

e.van_duijn@lumc.nl

Received 27 November 2013 Revised 19 March 2014 Accepted 24 March 2014

Published Online First

14 May 2014

\section{(a) CrossMark}

To cite: van Duijn $\mathrm{E}_{\text {, }}$ Craufurd D, Hubers AAM et al. I Neurol Neurosurg Psychiatry 2014;85: 1411-1418.

\section{ABSTRACT}

Background The majority of Huntington's disease (HD) mutation carriers experience some psychopathology during their lifetime, varying from irritability to psychosis, but prevalences of particular symptoms vary widely due to diverse study populations in different stages of HD and the use of different assessment methods.

Methods The study population consisted of 1993 HD mutation carriers from 15 European countries, all participating in the observational REGISTRY study. The behavioural section of the Unified HD Rating Scale was used to examine the prevalence and correlates of five neuropsychiatric features: depression, irritability/ aggression, obsessive/compulsive behaviours, apathy and psychosis.

Results Twenty-seven per cent of the participants did not have any neuropsychiatric symptom in the last month. Moderate to severe apathy occurred in $28.1 \%$ of the participants, whereas moderate to severe depression was found in $12.7 \%$. Irritable/aggressive symptoms were present in $13.9 \%$ of the participants, and $13.2 \%$ showed obsessive/compulsive behaviours. Moderate to severe psychotic symptoms were found in only $1.2 \%$. Only $54.9 \%$ of all participants with moderate to severe depression used antidepressants, suggesting undertreatment of depression. Obsessive/compulsive behaviours and irritability/aggression were inversely correlated with the Total Functional Capacity score, but with apathy showing the strongest inverse association. Conclusions A variety of neuropsychiatric symptoms are highly prevalent in different stages of HD in this European HD population, with apathy as the most frequent symptom. Depression, irritability/aggression and $\mathrm{OCBs}$ are prevalent in all stages of HD. Apathy was the key neuropsychiatric symptom occurring most often in advanced HD stages. Due to possible selection of relatively healthy participants, prevalences reported in this study might be an underestimation of prevalence in the entire $\mathrm{HD}$ population.

\section{INTRODUCTION}

Huntington's disease (HD) is an autosomaldominant neurodegenerative disease with psychiatric symptoms, cognitive deterioration, progressive motor abnormalities, ${ }^{1}$ and other physical symptoms like muscle atrophy and weight loss. ${ }^{2}$ HD results from an expanded trinucleotide CAG repeat sequence in the huntingtin (HTT) gene on chromosome $4{ }^{3} \mathrm{HD}$ typically has a mid-adult onset and a disease duration of 15-20 years. Prevalence of HD is $10-12$ per 100000 persons in the Western world, with many more people at risk of the disease. ${ }^{4}$ Although symptomatic treatment has improved significantly as a result of increased awareness of non-motor symptoms, no diseasemodifying treatment is available.

Reported prevalence rates of psychiatric symptoms and disorders vary widely due to diverse study populations in different stages of HD and the use of different assessment methods with varying definitions of psychiatric phenomena. ${ }^{5-10}$ Nevertheless, the majority of mutation carriers experience some psychopathology during their lifetime. Especially in the early stages of the disease, psychiatric symptoms are not always recognised as signs of HD, although they are significantly more prevalent in premotor symptomatic mutation carriers compared to noncarriers. ${ }^{8} 11$ In a large ongoing multinational observational study of HD (REGISTRY) in Europe, 87\% of the mutation carriers showed a form of behavioural disturbance. The investigators estimated that $20 \%$ had a psychiatric onset of the disease, whereas $39 \%$ had a lifetime history of 'severe psychiatric signs'. ${ }^{12}$

The assessment of formal psychiatric diagnoses in HD is complicated by the co-occurrence of cognitive, motor and physical symptoms. Therefore, the criteria of the Diagnostic Statistical Manual (DSM) of mental diseases ${ }^{13}$ may not be applicable to the behavioural phenomena observed in patients with progressive HD (or neurodegenerative diseases in general).

In this study, we aimed to assess the occurrence and correlates of common neuropsychiatric symptoms including depression, irritability/aggression, obsessive/compulsive behaviours, apathy and psychosis, using the behavioural section of the Unified Huntington's Disease Rating Scale (UHDRS-b) ${ }^{14}$ in the aforementioned REGISTRY study.

\section{MATERIAL AND METHODS \\ Participants}

The study population consisted of 1993 HD mutation carriers, all participating in the REGISTRY study of the European Huntington's Disease Network (EHDN) prior to February 2011. REGISTRY is a large, prospective study observing the natural course, clinical spectrum and management of HD in European countries. ${ }^{15}$ More 
information on the REGISTRY study can be found at (http:// www.euro-hd.net/html/registry).

In our study, mutation carriers in various disease stages (premanifest and manifest) from 15 European countries participated: Austria $(n=58)$, Belgium $(n=3)$, Czech Republic $(n=29)$, Finland $(n=19)$, France $(n=158)$, Germany $(n=492)$, Italy $(n=179)$, The Netherlands $(n=203)$, Norway $(n=74)$, Poland $(n=219)$, Portugal $(n=64)$, Spain $(n=159)$, Sweden $(n=15)$, Switzerland $(n=21)$, and the UK $(n=300)$. Only monitored data of REGISTRY participants who had a complete UHDRS behavioural assessment were included in our study.

Full ethical approval for the REGISTRY study was obtained in each of the participating countries, and all participants gave written informed consent.

\section{Sociodemographic and clinical characteristics}

Data on sociodemographic and clinical characteristics, including psychiatric history and use of psychotropic medication, were collected by trained interviewers using detailed electronic case report forms. The estimated duration of disease was calculated by the date of visit minus the rater-estimated date of disease onset. The Total Motor Score (TMS, range 0-124 points) of the UHDRS was used to assess motor functioning, with higher scores meaning more motor symptoms. ${ }^{14}$ Global functioning and disease stage were assessed using the Total Functional Capacity (TFC) scale of the UHDRS, with lower scores indicating worse global functioning (range $0-13$ points). ${ }^{16}$

\section{Behavioural subscales}

The UHDRS-b assesses frequency and severity of 11 neuropsychiatric symptoms in the month preceding the interview, with severity and frequency scores ranging from 0 (absent/ never) through 4 (severe/often). Total scores for the separate items of the UHDRS-b are computed by multiplying severity and frequency scores (range $0-16$ points). In this study, we used the item clusters that were previously reported in another study after a principal-components analysis. ${ }^{17}$ Four clusters were identified: depression, irritability/aggression, executive dysfunction and psychosis. However, the executive dysfunction cluster included obsessive/compulsive behaviours and apathy, which are clinically different neuropsychiatric features. Therefore, we defined five subscales for this study: depression, irritability/ aggression, obsessive/compulsive behaviours, apathy and psychosis. Total scores for each subscale are computed by summing the total scores of the separate items of each subscale. The depression subscale (range 0-64 points) consisted of four items: 'depressed mood', 'low self-esteem', 'suicidal ideation' and 'anxiety'. Participants were divided into three groups, depending on their total subscale score: no symptoms ( $\leq 4$ points), mild symptoms (5-16 points), or moderate to severe symptoms ( $>16$ points). The irritability/aggression subscale (range $0-32$ points) consisted of two items: 'irritability' and 'aggression'. Participants were divided into three groups, depending on their total subscale score: no symptoms ( $\leq 2$ points), mild symptoms (3-8 points), or moderate to severe symptoms ( $>8$ points). The obsessive/compulsive behaviours (OCBs) subscale (range 0-32 points) consisted of two items: 'perseveration' and 'compulsions'. Participants were divided into three groups, depending on their total subscale score: no symptoms ( $\leq 2$ points), mild symptoms (3-8 points), or moderate to severe symptoms ( $>8$ points). The apathy subscale (range $0-16$ points) consisted of one item: 'apathy'. Participants were divided into three groups, depending on their total subscale score: no symptoms $(\leq 1$ point), mild symptoms (2-4 points), or moderate to severe symptoms ( $>4$ points). The psychosis subscale (range 0-32 points) consisted of two items: 'delusions' and 'hallucinations'. Participants were divided into three groups, depending on their total subscale score: no symptoms ( $\leq 2$ points), mild symptoms (3-8 points), or moderate to severe symptoms ( $>8$ points).

\section{Statistical analyses}

Data are presented as $\mathrm{n}(\%)$, mean $( \pm \mathrm{SD})$, or median (IQR) when appropriate. Correlations between TFC score and disease duration, and between the different behavioural subscales were analysed using Spearman's rank order correlation. A $\chi^{2}$ test for linear-by-linear association was used to compare the prevalence of moderate to severe behavioural subscale scores across the different disease stages. Correlates of the behavioural subscale categories (no, mild and moderate to severe symptoms) were analysed using $\chi^{2}$ tests for categorical data, by analysis of variance for continuous variables with normal distributions, or nonparametric Kruskal-Wallis tests for continuous variables without normal distributions. Posthoc comparisons were done by $\chi^{2}$ tests for categorical data, Tukey honestly significant difference (HSD) test for independent samples with normal distributions, or nonparametric Kruskal-Wallis test. The independent correlates of each subscale were determined by comparing mutation carriers without subscale symptoms (ie, reference) with mutation carriers with moderate to severe subscale symptoms using multivariate logistic regression analysis. Variables with a p value $\leq 0.05$ in the univariate analyses and the variables, sex and age, were included in these multivariate analyses. Due to the low prevalence of the psychosis subscale, its correlates were determined by comparing 'no psychosis' with 'mild to severe psychosis' by $\chi^{2}$ tests for categorical data, by independent samples $t$ test for independent samples with normal distributions, or non-parametric KruskalWallis test for continuous variables without normal distributions. The independent correlates were determined by multivariate logistic regression analysis. Variables with a $\mathrm{p}$ value $\leq 0.05$ in the univariate analyses and the variables, sex and age, were included in the final multivariate model.

A $p$ value $<0.05$ was considered statistically significant. SPSS V.20.0 was used.

\section{RESULTS}

\section{Sociodemographic and clinical characteristics}

Of the 1993 mutation carriers, 51.1\% were male. The mean age of all mutation carriers was 50.3 years (SD: 12.3 ), and their estimated median disease duration was 5.9 years (IQR: 3.4-9.9). The mean TMS was 35.3 points (SD: 19.8). The study cohort consisted of mutation carriers from all TFC stages, but most mutation carriers were in the early and mid-disease stages. The correlation between TFC score and disease duration was -0.48 $(\mathrm{p}<0.001)$. Psychotropics were used by 1117 (56.0\%) mutation carriers (table 1 ).

Of all mutation carriers, $539(27.0 \%)$ had a score of 0 on all five behavioural subscales in the last month. The distinctive subscale scores had strong right-skewed distributions. Since the five behavioural subscales were relatively independent (all $r<0.40$ ), we analysed each behavioural subscale separately.

\section{Depression}

The median depression subscale score was 3 points (IQR: 0-10) with 1153 (57.9\%) mutation carriers scoring 'no depression', 586 (29.4\%) scoring 'mild depression', and 254 (12.7\%) scoring 'moderate to severe depression' (table 2). In disease Stage $1,10 \%$ of the mutation carriers had moderate to severe depression, which increased to $17.2 \%$ in Stage 4-5 (figure 1). 
Table 1 Characteristics and behavioural subscale scores in HD mutation carriers of the REGISTRY study

REGISTRY participants $(n=1993)$

\begin{tabular}{|c|c|}
\hline \multicolumn{2}{|l|}{ Sociodemographics } \\
\hline Sex, male & $1019(51.1)$ \\
\hline Age, years & $50.3 \pm 12.3$ \\
\hline \multicolumn{2}{|l|}{ Clinical characteristics } \\
\hline CAG repeat length, number & $44.6 \pm 4.3$ \\
\hline Duration of disease, years* & $5.9(3.4-9.9$ \\
\hline TMS, points & $35.3 \pm 19.8$ \\
\hline TFC, points & $8(5-12)$ \\
\hline \multicolumn{2}{|l|}{ Disease stage } \\
\hline Stage 1 & $662(33.2)$ \\
\hline Stage 2 & $655(32.9)$ \\
\hline Stage 3 & $513(25.7)$ \\
\hline Stage 4 & $140(7.0)$ \\
\hline Stage 5 & $23(1.2)$ \\
\hline \multicolumn{2}{|l|}{ Psychotropic medication use } \\
\hline Any psychotropicst & $1117(56.0)$ \\
\hline Benzodiazepinesł & $338(17.0)$ \\
\hline Antidepressants§ & $692(34.7)$ \\
\hline Antipsychotics & $679(34.1)$ \\
\hline Mood stabilisers/antiepileptics** & $144(7.2)$ \\
\hline \multicolumn{2}{|l|}{ Behavioural subscale scores } \\
\hline Depression, points & $3(0-10)$ \\
\hline Irritability/aggression, points & $1(0-5)$ \\
\hline OCBs, points & $0(0-3)$ \\
\hline Apathy, points & $1(0-6)$ \\
\hline Psychosis, points & $0(0-0)$ \\
\hline
\end{tabular}

Data are presented as $n(\%)$, mean $( \pm S D)$ or median $(I Q R)$ when appropriate.

*Duration of disease is calculated by date of visit minus the rater-estimated date of

disease onset.

†111 missings.

$\$ 78$ missings.

$\S 85$ missing.

१69 missings.

**19 missings due to missing values for use of psychotropics.

HD, Huntington's disease; OCBs, obsessive/compulsive behaviours; TFC, Total

Functional Capacity; TMS, Total Motor Score.

Mutation carriers with depression were more often female, had a lower TFC score, more often a positive psychiatric history for depression, obsessive/compulsive behaviours (OCB) and a suicide attempt, and used benzodiazepines, antidepressants and antipsychotics more often.

Independent correlates of depression were: a positive psychiatric history for depression $(p<0.001)$, OCBs $(p=0.001)$, a previous suicide attempt $(p=0.01)$, use of benzodiazepines $(p=0.004)$ and use of antidepressants $(p=0.046)($ table 2$)$.

\section{Irritability/aggression}

The median irritability/aggression subscale score was 1 point (IQR: 0-5) with $1223(61.4 \%)$ mutation carriers scoring 'no irritability/aggression', 493 (24.7\%) scoring 'mild irritability/ aggression', and 277 (13.9\%) scoring 'moderate to severe irritability/aggression' (table 3). The prevalence of moderate to severe irritability/aggression increased as disease stage increased, from $10.4 \%$ in Stage 1 to $19.6 \%$ in Stages 4-5 (figure 1).

Mutation carriers with irritability/aggression were more often male, had a longer duration of disease, a higher TMS, a lower TFC score, more often a positive psychiatric history for depression, OCBs, psychosis and a suicide attempt, and more often used antipsychotics and mood stabilisers/antiepileptics.
Independent correlates of irritability/aggression were: male sex $(p=0.01)$, younger age $(p=0.02)$, a positive psychiatric history for depression $(p=0.001)$, psychosis $(p=0.04)$ and a previous suicide attempt $(\mathrm{p}=0.02)$ (table 3$)$.

\section{OCBS}

The median OCBs subscale score was 0 points (IQR: $0-3$ ) with 1478 (74.2\%) mutation carriers scoring 'no OCBs', 252 (12.6\%) mutation carriers scoring 'mild OCBs', and 263 (13.2\%) scoring 'moderate to severe OCBs' (table 4). The prevalence of moderate to severe OCBs increased progressively as disease stage increased, from $4.5 \%$ in Stage 1 to $25.8 \%$ in Stages 4-5 (figure 1).

Mutation carriers with OCBs had a longer duration of disease, a higher TMS, a lower TFC score, more often a positive psychiatric history for depression, OCBs, psychosis and a previous suicide attempt, and more often used benzodiazepines, antidepressants, antipsychotics, and mood stabilisers/antiepileptics.

Independent correlates of OCBs were: a higher TMS $(p=0.02)$, a positive psychiatric history for depression $(p=0.01)$ and OCBs $(p<0.001)$, and use of benzodiazepines $(p=0.01)$ (table 4).

\section{Apathy}

The median apathy subscale score was 1 point (IQR: 0-6) with 1048 (52.6\%) mutation carriers scoring 'no apathy', 385 (19.3\%) mutation carriers scoring 'mild apathy', and 560 (28.1\%) scoring 'moderate to severe apathy' (table 5). The prevalence of moderate to severe apathy increased progressively as disease stage increased, from $11.8 \%$ in Stage 1 to $54.6 \%$ in Stages 4-5 (figure 1).

Mutation carriers with apathy were older, had a longer duration of disease, a higher TMS, a lower TFC score, more often a positive psychiatric history for depression, OCBs, psychosis and a previous suicide attempt, and more often used benzodiazepines, antidepressants, antipsychotics, and mood stabilisers/ antiepileptics.

Independent correlates of apathy were: male sex $(p=0.004)$, a lower TFC score $(p<0.001)$, a positive psychiatric history for depression $(p<0.001)$ and OCBs $(p=0.002)$, a previous suicide attempt $(\mathrm{p}=0.006)$, use of benzodiazepines $(\mathrm{p}=0.001)$, and use of antipsychotics $(\mathrm{p}=0.006)$ (table 5).

\section{Psychosis}

The median psychosis subscale score was 0 points (IQR: 0-0) with 1912 (95.9\%) mutation carriers scoring 'no psychosis', 58 (2.9\%) scoring 'mild psychosis', and 23 (1.2\%) scoring 'moderate to severe psychosis'. The highest prevalence of psychosis was in Stage $3(2.5 \%)$ (figure 1 ).

Since the number of mutation carriers with psychosis was very low, we analysed only two groups: 'no psychosis' $(n=1912)$ and 'mild to severe psychosis' $(n=81)$, and found that mutation carriers with psychosis had a significantly longer duration of disease, a higher TMS, a lower TFC score, more often a positive psychiatric history for depression, OCBs and psychosis, and more often used benzodiazepines and antipsychotics.

The only independent correlates of psychosis were a history of psychosis $(p<0.001)$ and use of benzodiazepines $(p=0.03)$ (table 6).

\section{DISCUSSION}

This large international study confirmed that depression, irritability/aggression, OCBs, and apathy are highly prevalent 
Table 2 Characteristics and correlates of depression subscale categories in HD mutation carriers

\begin{tabular}{|c|c|c|c|c|c|c|}
\hline & \multicolumn{4}{|c|}{ Univariate analyses } & \multicolumn{2}{|c|}{ Multivariate logistic regression } \\
\hline & No $(n=1153)$ & Mild $(n=586)$ & Moderate to severe $(n=254)$ & $\mathrm{p}$ Value & OR $(95 \% \mathrm{Cl})$ & p Value \\
\hline \multicolumn{7}{|l|}{ Sociodemographics } \\
\hline Sex, male & $635(55.1)^{*}$ & $277(47.3) \dagger$ & $107(42.1) \dagger$ & $<0.001$ & 0.74 (0.54 to 1.02$)$ & 0.06 \\
\hline Age, years (OR per 10 years) & $50.5 \pm 12.6$ & $50.0 \pm 11.8$ & $50.5 \pm 11.7$ & 0.70 & $1.03(0.90$ to 1.18$)$ & 0.68 \\
\hline \multicolumn{7}{|l|}{ Clinical characteristics } \\
\hline CAG repeats, number & $44.7 \pm 4.32$ & $44.5 \pm 4.25$ & $44.1 \pm 4.07$ & 0.12 & & \\
\hline Duration of disease, years & $5.9(3.2-9.9)$ & $5.9(3.5-9.6)$ & $6.2(3.5-10.6)$ & 0.53 & & \\
\hline TMS, points & $35.3 \pm 20.3$ & $36.2 \pm 19.3$ & $33.3 \pm 19.1$ & 0.16 & & \\
\hline TFC, points & $9(6-12)^{*}$ & $8(5-11) \dagger$ & $8(5-11) \dagger$ & 0.004 & 1.03 (0.98 to 1.08$)$ & 0.26 \\
\hline \multicolumn{7}{|l|}{ Psychiatric history } \\
\hline Depression & $416(36.1)^{*}$ & $359(61.3) \dagger$ & 209 (82.3)‡ & $<0.001$ & 5.57 (3.80 to 8.16$)$ & $<0.001$ \\
\hline OCBs & $71(6.2)^{*}$ & $79(13.5) \dagger$ & $52(20.5) \ddagger$ & $<0.001$ & 2.18 (1.38 to 3.42$)$ & 0.001 \\
\hline Psychosis & $70(6.1)$ & $47(8.0)$ & $21(8.3)$ & 0.21 & & \\
\hline Suicide attempt & $52(4.5)^{*}$ & $62(10.6) \dagger$ & $40(15.7) \ddagger$ & $<0.001$ & 2.01 (1.21 to 3.32$)$ & 0.01 \\
\hline \multicolumn{7}{|l|}{ Psychotropic medication use } \\
\hline Benzodiazepines & $144(12.9)^{*}$ & $125(22.2) \dagger$ & $69(28.9) \ddagger$ & $<0.001$ & $1.78(1.20$ to 2.62$)$ & 0.004 \\
\hline Antidepressants & $324(29.3)^{*}$ & $234(41.8) \dagger$ & $134(54.9) \ddagger$ & $<0.001$ & $1.42(1.01$ to 1.99$)$ & 0.046 \\
\hline Antipsychotics & $354(31.8)^{*}$ & $214(37.9) \dagger$ & $111(44.9) \dagger$ & $<0.001$ & $1.13(0.80$ to 1.61$)$ & 0.49 \\
\hline Mood stabilisers/antiepileptics & $70(6.1)$ & $50(8.7)$ & $24(9.5)$ & 0.06 & & \\
\hline
\end{tabular}

Bold represents the values with $p<0.05$

Univariate analyses: Data are presented as $n(\%)$, mean $( \pm$ SD) or median $(I Q R)$ when appropriate.

$\mathrm{p}$ Values by $\chi^{2}$ tests for categorical data, by one-way between-groups analysis of variance for independent samples with normal distribution, or non-parametric Kruskal-Wallis test for continuous variables without normal distributions. Values in the same row with different superscript letters are significantly different $(p<0.05)$.

Multivariate analyses: $\mathrm{OR}, 95 \% \mathrm{Cl}$ and $\mathrm{p}$ value by binary logistic regression.

Logistic regression analysis (enter model) for 'moderate to severe depression' ( $n=229)$ versus 'no depression' (ie, reference: $n=1065)$ (113 missings due to missing values (no start and stop date) for use of psychotropics). Covariates logistic regression analysis: All variables with $p$ value $<0.05$ in the univariate analysis were entered (Total Functional Capacity (TFC), depression in past, obsessive/compulsive behaviours (OCBs) in past, suicide attempt in past, use of benzodiazepines, use of antidepressants, use of antipsychotics), while sex and age were forced into the multivariate model.

HD, Huntington's disease; TMS, Total Motor Score.

neuropsychiatric symptoms in HD. By contrast, the prevalence of psychosis in this HD population with a median duration of disease of 5.9 years was low. Twenty-seven per cent of the mutation carriers did not have any neuropsychiatric symptom within the previous month of their visit to their physician.

Several cross-sectional studies previously reported that depressed mood and sadness are early symptoms of HD that peak during the early motor symptomatic phase, ${ }^{11} 18$ whereas significantly lower rates of depression are present in advanced stages of the disease. ${ }^{18}$ However, no independent correlation between depression on the one hand and duration of disease or TFC score on the other hand was found in this study. Although all neuropsychiatric symptoms were associated with the TFC score in univariate analyses, only apathy remained independently

Figure 1 Prevalences of moderate to severe symptoms on the behavioural subscales across different disease stages. Legend: Disease stages were defined according to the Total Functional Capacity (TFC) score: Stage 1=TFC score 13-11, Stage 2=TFC score 10-7, Stage $3=$ TFC score $6-3$, Stage $4=$ TFC score 2-1, Stage $5=$ TFC score 0 . Due to the low number of mutation carriers in Stage $5(n=23)$, Stages 4 and 5 were combined. ${ }^{*} p<0.05$, ${ }^{* *} p<0.01$

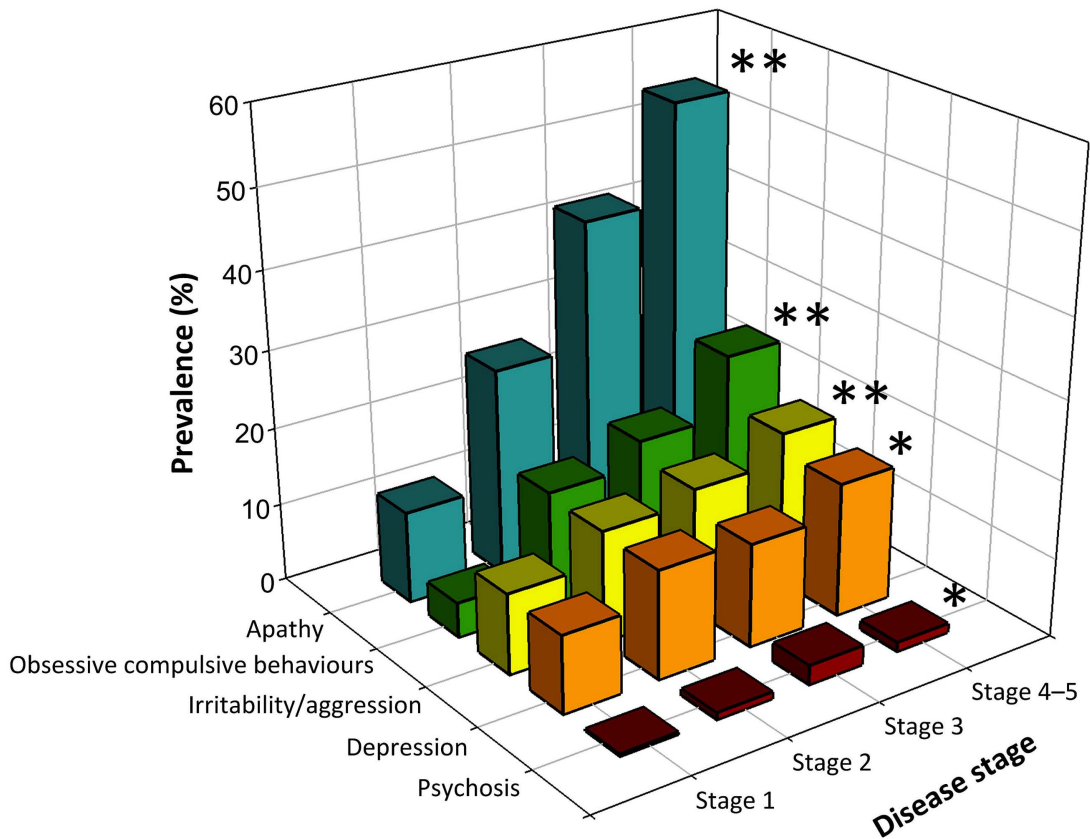


Table 3 Characteristics and correlates of irritability/aggression subscale categories in HD mutation carriers

\begin{tabular}{|c|c|c|c|c|c|c|}
\hline & \multicolumn{4}{|c|}{ Univariate analyses } & \multicolumn{2}{|c|}{ Multivariate logistic regression } \\
\hline & No $(n=1223)$ & Mild ( $n=493)$ & Moderate to severe $(n=277)$ & $\mathrm{p}$ Value & OR $(95 \% \mathrm{Cl})$ & $\mathrm{p}$ Value \\
\hline \multicolumn{7}{|l|}{ Sociodemographics } \\
\hline Sex, male & $625(51.1)$ & $235(47.7)^{*}$ & $159(57.4) \dagger$ & 0.04 & 1.45 (1.09 to 1.93$)$ & 0.01 \\
\hline Age, years (OR per 10 years) & $50.8 \pm 12.1$ & $49.8 \pm 12.4$ & $49.3 \pm 12.8$ & 0.12 & $0.86(0.76$ to 0.97$)$ & 0.02 \\
\hline \multicolumn{7}{|l|}{ Clinical characteristics } \\
\hline CAG repeats, number & $44.4 \pm 3.89$ & $44.7 \pm 4.93$ & $44.7 \pm 4.60$ & 0.34 & & \\
\hline Duration of disease, year & $5.7(3.3-9.6)^{*}$ & $6.0(3.5-9.8)$ & $6.3(3.5-10.6) \dagger$ & 0.05 & 1.01 (0.98 to 1.04$)$ & 0.47 \\
\hline TMS, points & $34.9 \pm 19.7^{*}$ & $34.6 \pm 19.6^{*}$ & $38.4 \pm 20.7 \dagger$ & 0.02 & 1.01 (1.00 to 1.02$)$ & 0.35 \\
\hline TFC, points & $9(5-12)^{*}$ & $9(6-12)^{*}$ & $7(5-11) \dagger$ & $<0.001$ & 0.97 (0.91 to 1.02$)$ & 0.23 \\
\hline \multicolumn{7}{|l|}{ Psychiatric history } \\
\hline Depression & $552(45.1)^{\star}$ & $264(53.5) \dagger$ & $168(60.6) \dagger$ & $<0.001$ & 1.70 (1.26 to 2.28$)$ & 0.001 \\
\hline OCBs & $94(7.7)^{*}$ & $65(13.2) \dagger$ & $43(15.5) \dagger$ & $<0.001$ & $1.48(0.96$ to 2.29$)$ & 0.08 \\
\hline Psychosis & $64(5.2)^{*}$ & $41(8.3) \dagger$ & $33(11.9) \dagger$ & $<0.001$ & 1.70 (1.03 to 2.82$)$ & 0.04 \\
\hline Suicide attempt & $83(6.8)^{*}$ & $32(6.5)^{*}$ & $39(14.1) \dagger$ & $<0.001$ & 1.71 (1.10 to 2.66$)$ & 0.02 \\
\hline \multicolumn{7}{|l|}{ Psychotropic medication use } \\
\hline Benzodiazepines & $206(17.5)$ & $75(15.5)$ & $57(22.1)$ & 0.08 & & \\
\hline Antidepressants & $417(35.7)$ & $168(35.4)$ & $107(40.1)$ & 0.38 & & \\
\hline Antipsychotics & $422(35.8)^{*}$ & $144(29.9) \dagger$ & $113(43.0) \ddagger$ & 0.002 & 0.97 (0.71 to 1.32$)$ & 0.85 \\
\hline Mood stabilisers/antiepileptics & $84(6.9)^{*}$ & $27(5.5)^{*}$ & $33(12.0) \dagger$ & 0.003 & 1.32 (0.83 to 2.10$)$ & 0.24 \\
\hline \multicolumn{7}{|c|}{$\begin{array}{l}\text { Bold represents the values with } p<0.05 .{ }^{*},+ \text { and } \neq: \text { Values in the same row with different superscript symbols are significantly different: } p<0.05 \text { (post hoc test). } \\
\text { Univariate analyses: Data are presented as } n(\%) \text {, mean }( \pm S D) \text { or median (IQR) when appropriate. } \\
p \text { Values by } \chi^{2} \text { tests for categorical data, by one-way between-groups analysis of variance for independent samples with normal distribution, or non-parametric Kruskal-Wallis test for } \\
\text { continuous variables without normal distributions. Values in the same row with different superscript letters are significantly different }(p<0.05) \text {. } \\
\text { Multivariate analyses: } O R, 95 \% C \text {, and } p \text { value by binary logistic regression. } \\
\text { Logistic regression analysis (enter model) for 'moderate to severe irritability/aggression' ( } n=261) \text { versus 'no irritability/aggression' (ie, reference: } n=1174) \text { ( } 65 \text { missings due to missing } \\
\text { values (no start and stop date) for use of psychotropics). Covariates logistic regression analysis: All variables with } p \text { value }<0.05 \text { in the univariate analysis were entered (duration of } \\
\text { disease, Total Motor Score (TMS), Total Functional Capacity (TFC), depression in past, obsessive/compulsive behaviours (OCBs) in past, psychosis in past, suicide attempt in past, use of } \\
\text { antipsychotics, use of mood stabilisers/antiepileptics), while sex and age were forced into the multivariate model. } \\
\text { HD, Huntington's disease. }\end{array}$} \\
\hline
\end{tabular}

Table 4 Characteristics and correlates of obsessive/compulsive behaviours (OCBs) subscale categories in HD mutation carriers

\begin{tabular}{|c|c|c|c|c|c|c|}
\hline & \multicolumn{4}{|c|}{ Univariate analyses } & \multicolumn{2}{|c|}{ Multivariate logistic regression } \\
\hline & No $(n=1478)$ & Mild ( $n=252)$ & Moderate to severe $(n=263)$ & $\mathrm{p}$ Value & OR $(95 \% \mathrm{Cl})$ & p Value \\
\hline \multicolumn{7}{|l|}{ Sociodemographics } \\
\hline Sex, male & 763 (51.6) & $123(48.8)$ & $133(50.6)$ & 0.70 & 1.13 (0.81 to 1.57$)$ & 0.47 \\
\hline Age, years (OR per 10 years) & $50.2 \pm 12.5$ & $50.2 \pm 11.8$ & $51.1 \pm 11.2$ & 0.58 & $0.99(0.86$ to 1.13$)$ & 0.83 \\
\hline \multicolumn{7}{|l|}{ Clinical characteristics } \\
\hline CAG repeats, number & $44.5 \pm 4.16$ & $44.9 \pm 4.50$ & $44.7 \pm 4.65$ & 0.24 & & \\
\hline Duration of disease, years & $5.6(3.2-9.4)^{*}$ & $6.0(3.6-10.0)^{*}$ & $8.0(4.8-12.0) \dagger$ & $<0.001$ & 1.01 (0.98 to 1.04$)$ & 0.61 \\
\hline TMS, points & $33.4 \pm 19.2^{*}$ & $38.2 \pm 20.6 \dagger$ & $43.5 \pm 20.3 \ddagger$ & $<0.001$ & 1.02 (1.01 to 1.03$)$ & 0.005 \\
\hline TFC, points & $9(6-12)^{*}$ & $8(5-11) \dagger$ & $6(4-9) \ddagger$ & $<0.001$ & 0.94 (0.88 to 1.01$)$ & 0.08 \\
\hline \multicolumn{7}{|l|}{ Psychiatric history } \\
\hline Depression & $666(45.1)^{*}$ & $138(54.8) \dagger$ & $180(68.4) \ddagger$ & $<0.001$ & 1.59 (1.10 to 2.28$)$ & 0.01 \\
\hline OCBs & $52(3.5)^{*}$ & $44(17.5) \dagger$ & $106(40.3) \ddagger$ & $<0.001$ & 14.46 (9.38 to 22.27$)$ & $<0.001$ \\
\hline Psychosis & $70(4.7)^{*}$ & $24(9.5) \dagger$ & $44(16.7) \ddagger$ & $<0.001$ & 1.11 (0.61 to 2.03$)$ & 0.73 \\
\hline Suicide attempt & $101(6.8)^{*}$ & $23(9.1)$ & $30(11.4) \dagger$ & 0.03 & $1.04(0.59$ to 1.85$)$ & 0.90 \\
\hline \multicolumn{7}{|l|}{ Psychotropic medication use } \\
\hline Benzodiazepines & $208(14.6)^{*}$ & $57(23.4) \dagger$ & $73(29.7) \dagger$ & $<0.001$ & 1.67 (1.13 to 2.48$)$ & 0.01 \\
\hline Antidepressants & $470(33.2)^{*}$ & $98(40.3) \dagger$ & $124(49.4) \ddagger$ & $<0.001$ & 1.32 (0.93 to 1.89$)$ & 0.12 \\
\hline Antipsychotics & $486(34.0)^{*}$ & $79(32.5)^{*}$ & $114(45.2) \dagger$ & 0.002 & 0.75 (0.51 to 1.08$)$ & 0.13 \\
\hline Mood stabilisers/antiepileptics & $98(6.7)^{*}$ & $13(5.2)^{*}$ & $33(12.8) \dagger$ & 0.001 & 1.16 (0.67 to 2.02$)$ & 0.60 \\
\hline \multicolumn{7}{|c|}{$\begin{array}{l}\text { Bold represents the values with } p<0.05 .{ }^{*},+ \text { and } \neq: \text { Values in the same row with different superscript symbols are significantly different: } p<0.05 \text { (post hoc test). } \\
\text { Univariate analyses: Data are presented as } n(\%) \text {, mean }( \pm S D) \text { or median (IQR) when appropriate. } \\
p \text { Values by } \chi^{2} \text { tests for categorical data, by one-way between-groups analysis of variance for independent samples with normal distribution, or non-parametric Kruskal-Wallis test for } \\
\text { continuous variables without normal distributions. Values in the same row with different superscript letters are significantly different ( } p<0.05) \text {. } \\
\text { Multivariate analyses: } O R, 95 \% C \text {, and p value by binary logistic regression. } \\
\left.\text { Logistic regression analysis (enter model) for 'moderate to severe } O C B s^{\prime}(n=232) \text { versus 'no } 0 C B s^{\prime} \text { (ie, reference: } n=1364\right)(145 \text { missings due to missing values (no start and stop date) } \\
\text { for use of psychotropics). Covariates logistic regression analysis: All variables with } p \text { value }<0.05 \text { in the univariate analysis were entered (duration of disease, Total Motor } S c o r e(T M S) \text {, } \\
\text { Total Functional Capacity (TFC), depression in past, obsessive/compulsive behaviours (OCBs) in past, psychosis in past, suicide attempt in past, use of benzodiazepines, use of } \\
\text { antidepressants, use of antipsychotics, use of mood stabilisers/antiepileptics), while sex and age were forced into the multivariate model. } \\
H D \text {, Huntington's disease. }\end{array}$} \\
\hline
\end{tabular}


Table 5 Characteristics and correlates of apathy subscale categories in HD mutation carriers

\begin{tabular}{|c|c|c|c|c|c|c|}
\hline & \multicolumn{4}{|c|}{ Univariate analyses } & \multicolumn{2}{|c|}{ Multivariate logistic regression } \\
\hline & No $(n=1048)$ & Mild ( $n=385)$ & $\begin{array}{l}\text { Moderate to } \\
\text { severe }(n=560)\end{array}$ & $\mathrm{p}$ Value & OR (95\% Cl) & $p$ Value \\
\hline \multicolumn{7}{|l|}{ Sociodemographics } \\
\hline Sex, male & $528(50.4)$ & $189(49.1)$ & $302(53.9)$ & 0.27 & $1.44(1.12$ to 1.85$)$ & 0.004 \\
\hline Age, years (OR per 10 years) & $49.4 \pm 12.3^{*}$ & $50.2 \pm 11.8^{*}$ & $52.2 \pm 12.4 \dagger$ & $<0.001$ & 1.09 (0.98 to 1.21$)$ & 0.11 \\
\hline \multicolumn{7}{|l|}{ Clinical characteristics } \\
\hline CAG repeats, number & $44.7 \pm 4.30$ & $44.4 \pm 3.95$ & $44.4 \pm 4.42$ & 0.42 & & \\
\hline Duration of disease, years & $5.3(2.9-8.8)^{*}$ & $6.2(3.4-9.8) \dagger$ & $7.3(4.6-11.2) \ddagger$ & $<0.001$ & $1.00(0.98$ to 1.03$)$ & 0.81 \\
\hline TMS, points & $31.9 \pm 19.0^{*}$ & $34.2 \pm 18.1^{*}$ & $42.5 \pm 20.7 \dagger$ & $<0.001$ & 1.00 (0.99 to 1.01$)$ & 0.63 \\
\hline TFC, points & $10(7-12)^{*}$ & $8(6-11) \dagger$ & $6(4-9) \ddagger$ & $<0.001$ & $0.81(0.77$ to 0.86$)$ & $<0.001$ \\
\hline \multicolumn{7}{|l|}{ Psychiatric history } \\
\hline Depression & $414(39.5)^{*}$ & $213(55.3) \dagger$ & $357(63.7) \ddagger$ & $<0.001$ & 2.06 (1.57 to 2.70$)$ & $<0.001$ \\
\hline OCBs & $66(6.3)^{*}$ & $45(11.7) \dagger$ & $91(16.2) \dagger$ & $<0.001$ & 1.96 (1.29 to 2.97$)$ & 0.002 \\
\hline Psychosis & $52(5.0)^{*}$ & $31(8.1) \dagger$ & $55(9.8) \dagger$ & 0.001 & $0.63(0.38$ to 1.06$)$ & 0.08 \\
\hline Suicide attempt & $56(5.3)^{*}$ & $29(7.5)^{*}$ & 69 (12.3)‡ & $<0.001$ & 1.89 (1.20 to 2.98$)$ & 0.006 \\
\hline \multicolumn{7}{|l|}{ Psychotropic medication use } \\
\hline Benzodiazepines & $121(11.9)^{*}$ & $73(19.6) \dagger$ & $144(27.2) \ddagger$ & $<0.001$ & 1.71 (1.24 to 2.37$)$ & 0.001 \\
\hline Antidepressants & $287(28.6)^{*}$ & $160(42.7) \dagger$ & $245(46.2) \dagger$ & $<0.001$ & 1.04 (0.79 to 1.37$)$ & 0.78 \\
\hline Antipsychotics & $265(26.0)^{*}$ & $150(39.8) \dagger$ & $264(50.1) \ddagger$ & $<0.001$ & 1.48 (1.12 to 1.94$)$ & 0.006 \\
\hline Mood stabilisers/antiepileptics & $60(5.8)^{*}$ & $28(7.4)^{*}$ & $56(10.1) \dagger$ & 0.006 & 0.84 (0.53 to 1.34$)$ & 0.47 \\
\hline \multicolumn{7}{|c|}{ 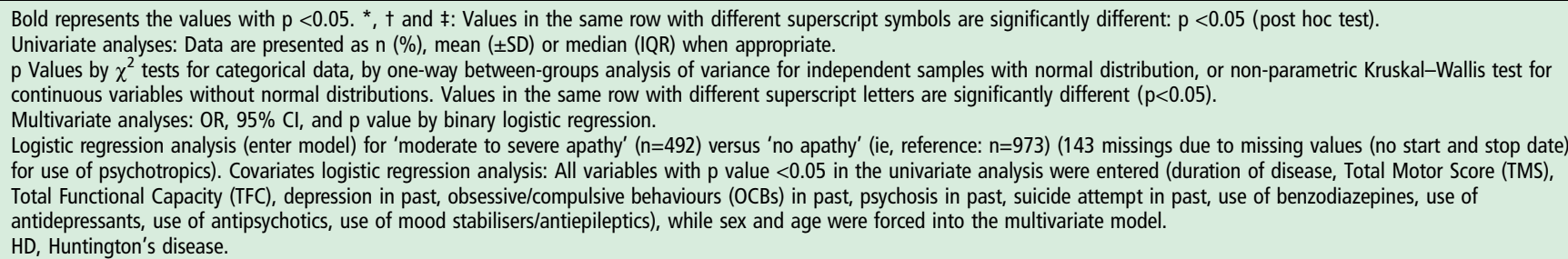 } \\
\hline
\end{tabular}

Table 6 Characteristics and correlates of psychosis subscale categories in HD mutation carriers

\begin{tabular}{|c|c|c|c|c|c|}
\hline & \multicolumn{3}{|c|}{ Univariate analyses } & \multicolumn{2}{|c|}{ Multivariate logistic regression } \\
\hline & No $(n=1912)$ & Mild to severe $(n=81)$ & p Vvalue & OR $(95 \% \mathrm{Cl})$ & p Value \\
\hline \multicolumn{6}{|l|}{ Sociodemographics } \\
\hline Sex, male & $975(51.0 \%)$ & $44(54.3 \%)$ & 0.56 & 1.45 (0.85 to 2.48$)$ & 0.17 \\
\hline Age, years (OR per 10 years) & $50.3 \pm 12.3$ & $51.0 \pm 12.3$ & 0.59 & $1.08(0.86$ to 1.35$)$ & 0.51 \\
\hline \multicolumn{6}{|l|}{ Clinical characteristics } \\
\hline CAG repeats, number & $44.5 \pm 4.22$ & $44.9 \pm 5.36$ & 0.40 & & \\
\hline Duration of disease, years & $5.8(3.3-9.8)$ & $7.3(5.3-11.4)$ & 0.001 & $1.00(0.94$ to 1.05$)$ & 0.85 \\
\hline TMS, points & $34.9 \pm 19.8$ & $43.5 \pm 20.3$ & $<0.001$ & 1.01 (0.99 to 1.03$)$ & 0.32 \\
\hline TFC, points & $9(6-12)$ & $6(4-8)$ & $<0.001$ & $0.94(0.85$ to 1.05$)$ & 0.28 \\
\hline \multicolumn{6}{|l|}{ Psychiatric history } \\
\hline Depression & $930(48.6)$ & $54(66.7)$ & 0.001 & $1.09(0.60$ to 2.00$)$ & 0.77 \\
\hline OCBs & $174(9.1)$ & $28(34.6)$ & $<0.001$ & $1.86(0.98$ to 3.55$)$ & 0.06 \\
\hline Psychosis & $97(5.1)$ & $41(50.6)$ & $<0.001$ & 14.98 (8.07 to 27.82 ) & $<0.001$ \\
\hline Suicide attempt & $144(7.5)$ & $10(12.3)$ & 0.11 & & \\
\hline \multicolumn{6}{|l|}{ Psychotropic medication use } \\
\hline Benzodiazepines & $313(17.0)$ & $25(35.2)$ & $<0.001$ & 1.90 (1.06 to 3.42$)$ & 0.03 \\
\hline Antidepressants & $669(36.5)$ & $23(30.3)$ & 0.27 & & \\
\hline Antipsychotics & 637 (34.5) & $42(54.5)$ & $<0.001$ & 0.84 (0.47 to 1.52$)$ & 0.57 \\
\hline Mood stabilisers/antiepileptics & $136(7.2)$ & $8(9.9)$ & 0.36 & & \\
\hline
\end{tabular}

Bold represents the values with $p<0.05$.

Univariate analyses: Data are presented as $n(\%)$, mean $( \pm$ SD) or median (IQR) when appropriate.

$\mathrm{p}$ Values by $\chi^{2}$ tests for categorical data, by independent samples $t$ test for independent samples with normal distribution, or non-parametric Kruskal-Wallis test for continuous variables without normal distributions.

Multivariate analyses: $\mathrm{OR}, 95 \% \mathrm{Cl}$, and $\mathrm{p}$ value by binary logistic regression.

Logistic regression analysis (enter model) for 'mild to severe psychosis' $(n=69)$ versus 'no psychosis' (ie, reference: $n=1797)$ (127 missings due to missing values (no start and stop date) for use of psychotropics). Covariates logistic regression analysis: All variables with p value $<0.05$ in the univariate analysis were entered (duration of disease, Total Motor Score (TMS), Total Functional Capacity (TFC), depression in past, obsessive/compulsive behaviours (OCBs) in past, psychosis in past, use of benzodiazepines, use of antipsychotics), while sex and age were forced into the multivariate model.

$H D$, Huntington's disease. 
inversely correlated with the TFC score after multivariate logistic regression analysis, indicating that apathy is more prevalent in advanced disease stages and may be the only neuropsychiatric symptom that is linearly related to progressive neurodegeneration.

Since our study used the UHDRS-b that asks about the presence of neuropsychiatric symptoms within the last month, our prevalence may differ from earlier studies. In general, one would expect higher prevalences when longer time periods are measured, although other methodological differences like varying cut-offs and different assessment tools complicate a comparison with the results of previous studies. ${ }^{19}$

The presence of all neuropsychiatric symptoms during the last month was associated with a positive psychiatric history, and a past episode of depression was particularly associated with all current neuropsychiatric symptoms, except psychosis. Psychosis in HD seems to occur as a rather isolated psychiatric phenomenon. We found a rather low prevalence of psychosis in our sample (4.1\%), which is in line with other studies that reported prevalences varying between $1 \%$ and $11 \% .^{5} 9^{20-23}$ Our estimate may, however, be low due to the young study population with a median disease duration of 5.9 years, since psychotic symptoms typically occur in advanced disease stages. ${ }^{24}$ The low prevalence of psychosis may also be explained by the relatively high use of antipsychotics, and to selection, since psychotic mutation carriers may have been less inclined to participate in REGISTRY.

As a result of the naturalistic design of our study, the use of medication has influenced our results. As expected, the use of antidepressants and benzodiazepines was correlated with depression. However, only $54.9 \%$ of all mutation carriers with moderate to severe depression used antidepressants, suggesting undertreatment of depressive symptoms. Alike, psychotic mutation carriers were undertreated as well with only $54.5 \%$ of the mildly to severely psychotic mutation carriers receiving antipsychotic medication. Apathy was independently associated with the use of antipsychotics and the use of benzodiazepines, however, we cannot disentangle whether the use of psychotropic medication is a cause or consequence of apathy. Since development of apathy as a side effect of the use of antipsychotics and benzodiazepines is very well possible, due to their blunting and sedative effects, we recommend to reduce the use of antipsychotics and benzodiazepines in apathetic patients.

Because of practical constraints for such a large-scale study with limited resources, analyses were done using naturalistic data with physicians collecting data as part of the 'real-world' setting of usual care. It therefore closely resembles the actual contexts and constraints faced by practitioners. The large number of participants with in-depth genetic, demographic and clinical assessments in the context of a standardised study protocol is a strength of this study. However, these data also carry some weaknesses. First, the naturalistic data is less rigorously collected than would have occurred in a prospective cohort study with measurements at regularly spaced intervals. Second, the generalisability of the results of this study might still be limited since the recruitment of participants differs between study sites. It is unclear how many of the eligible mutation carriers eventually entered the database. HD patients attending REGISTRY clinics might be less disturbed, in less advanced disease stages, and better monitored than the general HD population. Therefore, prevalences and independent correlates of the behavioural subscales described may be different for HD patients groups in a more advanced disease stage. For the same reason, we may have included fewer HD mutation carriers with severe behavioural symptoms, for example, psychosis, that may have prevented participation in REGISTRY. Third, confounding by indication precludes conclusions about the effectiveness of psychotropic medication in our participants. If the treatment has been effective, the frequent prescription of psychotropic medications could reflect a higher prevalence of behavioural symptoms prior to treatment. Additionally, when antipsychotics were prescribed as a treatment for motor symptoms, this may have suppressed psychotic symptoms or irritability in particular. Moreover, in this observational study, physicians have selected the treatments and, likewise, patients have had a saying in this, based on their own motivations. Fourth, a control group (eg, non-mutation carriers from HD families) was not included in this study design, so we cannot disentangle the effects of environmental factors, for example, living in an HD family, which may have contributed to behavioural symptoms. Finally, there are plausible alternative explanations for the neuropsychiatric symptoms, as there is likely residual and unmeasured confounding. Unmeasured characteristics that may have been of importance are recent life events, a lack of social support, and health-related behaviours. Thus, despite the fact that our results are based on analyses using a large cross-country database, our findings must be interpreted cautiously, and generally be viewed as hypothesis-generating.

We conclude that depression, irritability/aggression and OCBs are prevalent, distressing and often unresolved by treatment in all stages of HD. Apathy was the key neuropsychiatric symptom occurring most often in advanced HD stages. These symptoms are leading to important impairments in quality of life, and place a significant burden on healthcare services. As neuropsychiatric symptoms found in HD do not fit well with major depression and other DSM-IV diagnoses, it is important for physicians to specifically check these symptoms for early detection and a more successful management.

\section{Author affiliations}

${ }^{1}$ Department of Psychiatry, Leiden University Medical Centre, Leiden, The Netherlands

${ }^{2}$ Centre for Mental Health Care Delfland, Delft, The Netherlands

${ }^{3}$ Insitute of Human Development, University of Manchester, Manchester, UK

${ }^{4}$ Manchester Centre for Genomic Medicine, Central Manchester University Hospitals NHS Foundation Trust and Manchester Academic Health Sciences Centre,

Manchester, UK

${ }^{5}$ Sigmund Freud University, Vienna, Austria

${ }^{6}$ Department of Neuropsychiatry, Birmingham and Solihull Mental Health Foundation Trust, Birmingham, UK

${ }^{7}$ Department of Psychiatry, Georgetown University, Washington, DC, USA

${ }^{8}$ Research Centre of Habilitation and Rehabilitation Models and Services, Faculty of Medicine, Institute of Health and Society, University of Oslo, Oslo, Norway

${ }^{9}$ Division of Surgery and Clinical Neurosciences, Department of Neurohabilitation, Oslo University Hospital, Oslo, Norway

${ }^{10}$ Department of Neurology, University of Ulm, Ulm, Germany

\section{Competing interests None.}

Ethics approval All medical ethical committees of all participating institutions.

Provenance and peer review Not commissioned; externally peer reviewed.

Data sharing statement Data is available for all members of the European Huntington's Disease Network (http://www.ehdn.net).

\section{REFERENCES}

1 Walker FO. Huntington's disease. Lancet 2007;369:218-28.

2 van der Burgh JM, Björkqvist M, Brundin P. Beyond the brain: widespread pathology in Huntington's disease. Lancet Neurol 2009;8:765-74.

3 Huntington's Disease Collaborative Research Group. A novel gene containing a trinucleotide repeat that is expanded and unstable on Huntington's disease chromosomes. Cell 1993;72:971-83.

4 Evans SJW, Douglas I, Rawlins MD, et al. Prevalence of adult Huntington's disease in the UK based on diagnosis recorded in general practice records. J Neurol Neurosurg Psychiatry 2013:84:1156-60. 
5 Paulsen JS, Ready RE, Hamilton JM, et al. Neuropsychiatric aspects of Huntington's disease. J Neurol Neurosurg Psychiatry 2001;71:310-14.

6 Berrios GE, Wagle AC, Marková IS, et al. Psychiatric symptoms in neurologically asymptomatic Huntington's disease gene carriers: a comparison with gene negative at risk subjects. Acta Psychiatr Scand 2002;105:224-30.

7 van Duijn E, Kingma EM, van der Mast RC. Psychopathology in verified Huntington's disease gene carriers. J Neuropsychiatry Clin Neurosci 2007;19: 441-8.

8 Duff K, Paulsen JS, Beglinger LJ, et al. Psychiatric symptoms in Huntington's disease before diagnosis: the Predict-HD study. Biol Psychiatry 2007;62:1341-6.

9 Julien CL, Thompson JC, Wild S, et al. Psychiatric disorders in pre-clinical Huntington's disease. J Neurol Neurosurg Psychiatry 2007;78:939-43.

10 Thompson JC, Harris J, Sollom CL, et al. Longitudinal evaluation of neuropsychiatric symptoms in Huntington's disease. J Neuropsychiatry Clin Neurosci 2012;24: 53-60.

11 Gargiulo M, Lejeune S, Tanguy ML, et al. Long-term outcome of presymptomatic testing in Huntington disease. Eur J Hum Genet 2009;17:165-71.

12 Orth M, Handley OJ, Schwenke C, et al. Observing Huntington's disease: the European Huntington's Disease Network's REGISTRY. PLoS Current 2010. doi:10. 1371/currents.RRN1184

13 American Psychiatric Association. Diagnostic and statistical manual of mental disorders. 4th edn. Washington, DC: American Psychiatric Association, 2000.

14 Huntington Study Group: Unified Huntington's disease rating scale. reliability and consistency. Mov Disord 1996;11:136-42.
15 Handley 0 , van Walsem M, Juni $P$, et al. Study protocol of Registry version 2.0 European Huntington's Disease Network (EHDN). Hygeia Public Health 2011;46:115-82.

16 Shoulson I, Fahn S. Huntington disease: clinical care and evaluation. Neurology 1979:29:1-3.

17 Rickards H, De Souza J, van Walsem M, et al. Factor analysis of behavioural symptoms in Huntington's disease. J Neurol Neurosurg Psychiatry 2011:82:411-12.

18 Paulsen JS, Nehl C, Hoth KF, et al. Depression and stages of Huntington's disease. $J$ Neuropsychiatry Clin Neurosci 2005;17:496-502.

19 Rickards H, De Souza J, Crooks J, et al. Discriminant analysis of Beck Depression Inventory and Hamilton Rating Scale for depression in Huntington's disease. $J$ Neuropsychiatry Clin Neurosci 2011;23:399-402.

20 Kulisevsky J, Litvan I, Berthier ML, et al. Neuropsychiatric assessment of Gilles de la Tourette patients: comparative study with other hyperkinetic and hypokinetic movement disorders. Mov Disord 2001;16:1098-104.

21 Murgod UA, Saleem Q, Anand A, et al. A clinical study of patients with genetically confirmed Huntington's disease from India. J Neuro/ Sci 2001;190:73-8.

22 Craufurd D, Thompson JC, Snowden JS. Behavioural changes in Huntington Disease. Neuropsychiatry Neuropsychol Behav Neurol 2001;14:219-26.

23 van Duijn E, Kingma EM, Timman $R$, et al. Cross-sectional study on prevalences of psychiatric disorders in mutation carriers of Huntington's disease compared with mutation-negative first-degree relatives. J Clin Psychiatry 2008;69:1804-10.

24 Marshall J, White K, Weaver $\mathrm{M}$, et al. Specific psychiatric manifestations among preclinical Huntington disease mutation carriers. Arch Neurol 2007;64:116-21. 